\title{
Epidemiology and Pathophysiology of Intracranial Large Artery Stenosis
}

\author{
Yousef Mohammad*, Marwan Qattan and Shyam Prabhakaran \\ Department of Neurological Sciences, Rush University Medical Center, Chicago, IL 60612, USA
}

\begin{abstract}
Intracranial stenosis due to atherosclerosis is a disease of significant prevalence worldwide and is responsible for a significant economic burden due to stroke-related disability. Known risk factors including age, race, sex, and medical co-morbidities contribute to its incidence. While ischemic stroke and transient ischemic attack can develop from myriad mechanisms in patients with intracranial stenosis, it is likely that an interplay of hemodynamic failure, in-situ thrombosis, and distal embolism results in brain ischemia. Knowledge of disease prevalence, risk factors, and pathophysiology is critical to development of better medical and interventional treatments for this grave condition.
\end{abstract}

Keywords: Ischemic stroke, intracranial stenosis, risk factors.

\section{INCIDENCE AND PREVALENCE}

Worldwide, stroke is surpassed by only heart disease as a cause of death [1]. In the United States (US), it is third behind only heart disease and cancer in mortality [2]. It is, however, the leading cause of long term disability in adults. Annually, about 780,000 individuals have new or recurrent strokes in the US. This corresponds to one stroke every 40 seconds. As the population ages, the incidence is on the rise. In fact, it is projected that stroke incidence will reach 1 million annually by the year 2050. If developing countries adopt a "Western" diet, there may be further increase in the incidence and prevalence of cardiovascular diseases and stroke $[3,4]$.

The recent advances in diagnostic tools, such as magnetic resonance angiography (MRA), CT angiography, and transesophageal echocardiography, have established stroke as a heterogeneous disease with multiple distinct causative mechanisms. Identifying the responsible mechanism in an individual patient has crucial implications in relation to targeted treatment and prevention. For instance, if the mechanism of stroke is attributed to cardiac embolism from atrial fibrillation then Warfarin becomes the best strategy for preventing recurrent stroke. However, if the mechanism is attributed to severe internal carotid artery stenosis then carotid endarterectomy becomes the best approach for reducing the risk of recurrent stroke. Based on these and other observations, ischemic stroke is classified into five categories based on cause: a) cardiac embolism; b) large artery atherosclerotic disease (extra- or intra-cranial); c) small vessel disease; d) stroke of other determined cause such as a coagulopathy; and e) stroke of undetermined cause [5].

This review will focus on atherosclerotic, intracranial, large artery stenosis. The major vessels involved are the supraclinoid internal carotid artery (ICA), proximal middle

*Address correspondence to this author at the Department of Neurological Sciences, Rush University Medical Center, Chicago, IL 60612, USA; Tel: 312-942-4500; Fax: 312-563-2206; E-mail: yousef_mohammad@ rush.edu cerebral artery (MCA) and anterior cerebral artery (ACA), distal vertebral artery (VA), proximal to mid-basilar artery (BA) and proximal posterior cerebral artery (PCA). The MCA is the most commonly affected artery followed by ICA, VA and BA [6, 7]. The stem (M1) and its major superior branch (M2) are the most common sites for MCA disease. Intracranial VA disease is more common than BA involvement but less common than extracranial VA disease. As much as $20 \%$ of strokes caused by intracranial atherosclerosis occur in the vertebro-basilar (VB) circulation [8]. Of note, $39 \%$ of VA disease is bilateral with $24 \%$ and $36 \%$ of patients having associated BA and extracranial VA disease [9]. The supraclinoid ICA is the most common location in American white [4, 6]. In blacks and Hispanics, intracranial stenosis afflicts younger patients than it does in whites [10]. Women may be more likely to have posterior circulation disease than men [8].

The incidence and prevalence of the condition varies according to the population studied. It is less common in Northern Europeans and Americans of European descent and more common in Asians of China, Japan and Korea [6, 10]. In the United States (US), approximately $10 \%$ of all strokes are attributed to intracranial stenosis, 78,000 strokes a year $[11,12]$. However, even in the US, the incidence differs according the racial or ethnic group studied. Of all ischemic strokes intracranial stenosis is responsible for $6-10 \%$ in whites, $11 \%$ in Hispanics and 6-29\% in blacks [10]. Suri et al. reported an estimated prevalence of symptomatic intracranial stenosis of 1 in 100,000 whites and 15 in 100,000 blacks [13]. Based on the Northern Manhattan study, an even higher prevalence of symptomatic intracranial atherosclerosis was noted in blacks and Hispanics (4.4\%). In the Hispanics, $11 \%$ of all strokes were attributed to intracranial stenosis [10].

From Asian studies, intracranial stenosis is estimated to account for $33-50 \%$ of all strokes in Chinese patients; $47 \%$ of Thailand patients; $48 \%$ of Singapore patients; and 10-25\% of Korean patients $[14,15]$. In a study conducted on acute ischemic stroke patients in China, $33 \%$ of acute stroke was attributed to intracranial large artery stenosis [16]. In another 
study conducted in Korea utilizing transcranial Doppler (TCD) and or MRA, intracranial stenosis (symptomatic and asymptomatic) existed in $54 \%$ of subjects. Indeed, stroke is the leading cause of death in Korea and intracranial stenosis accounts for much of that public health burden [17].

These racial and ethnic differences have been demonstrated in many angiographic and autopsy studies (Table 1). Overall, the data consistently demonstrates that blacks, Asians, and Hispanics have more intracranial atherosclerotic disease in contrast to whites who have more extracranial carotid disease $[10,18-24]$. As Asians, Hispanics, and blacks constitute the majority of the world's population, it may be inferred that intracranial stenosis is the most common stroke mechanism worldwide. The stroke incidence is expected to grow as the above mentioned populations expand and modernization of lifestyle and diet ensue.

\section{RISK FACTORS}

Risk factors for intracranial stenosis may be categorized into three groups:

\section{Non-modifiable risk factors}

2. Modifiable risk factors

3. Less well documented risk factors

Among the many studies of prevalence and risk factors for intracranial stenosis, age, race, hypertension, and diabetes have been most strongly and consistently observed to be associated with the disease.

\section{Non-Modifiable Risk Factors}

\section{Race (Table 1)}

As demonstrated in the first section, intracranial stenosis is more common in African and Hispanic Americans compared to white Americans. The incidence is even much higher in the Asians population. For example, the relative risk of having intracranial stenosis is 5-fold greater in American blacks and 5.85-fold greater in Hispanics compared to American whites [13]. This is supported by data from the Northern Manhattan Stroke Study that showed the likelihood for American blacks and Hispanics to develop ischemic stroke was nearly 8 -fold higher than whites [10]. This racial difference in the prevalence of intracranial stenosis was also demonstrated in autopsy studies that found African Americans had intracranial atherosclerosis more frequently compared to whites [18]. Whites had more atherosclerosis in the aorta and coronary arteries. Another autopsy study showed African Americans had more disease in the MCA stem and supraclinoid ICA, whereas whites had more frequent disease at the origin of the ICA [19]. Furthermore, the Asian population is at increased risk for developing intracranial stenosis compared to subjects of Northern European descent. In fact, it is also the most common cerebrovascular lesion in the Asian population [15, 16].

Age

Age is an independent risk factor for the development of intracranial atherosclerosis. In a 1967 autopsy study by Baker et al. [25], the prevalence of intracranial atherosclerosis was demonstrated to increase with each decade of age. It was found in $23 \%$ of those $50-59$ years of age, $43 \%$ of those $60-69$ years of age, $65 \%$ of those $70-79$ years of age and $80 \%$ of those $>80$ years of age. An autopsy study by Solberg and McGarry found similar results with respect to age [18]. A study conducted in the South Korean population observed that for every 10 years of age, the odds of intracranial disease increased by 1.2 [26]. The impact of age on developing intracranial stenosis escalates even more in the presence of other risk factors such as diabetes mellitus hypertension and hyperlipidemia [7].

\section{Sex}

Intracranial stenosis is more common among men, particularly in younger age groups and in particular locations, such as the basilar artery. Cross-sectional population-based and autopsy-based epidemiological studies

Table 1. Racial Distribution of Intracranial Disease

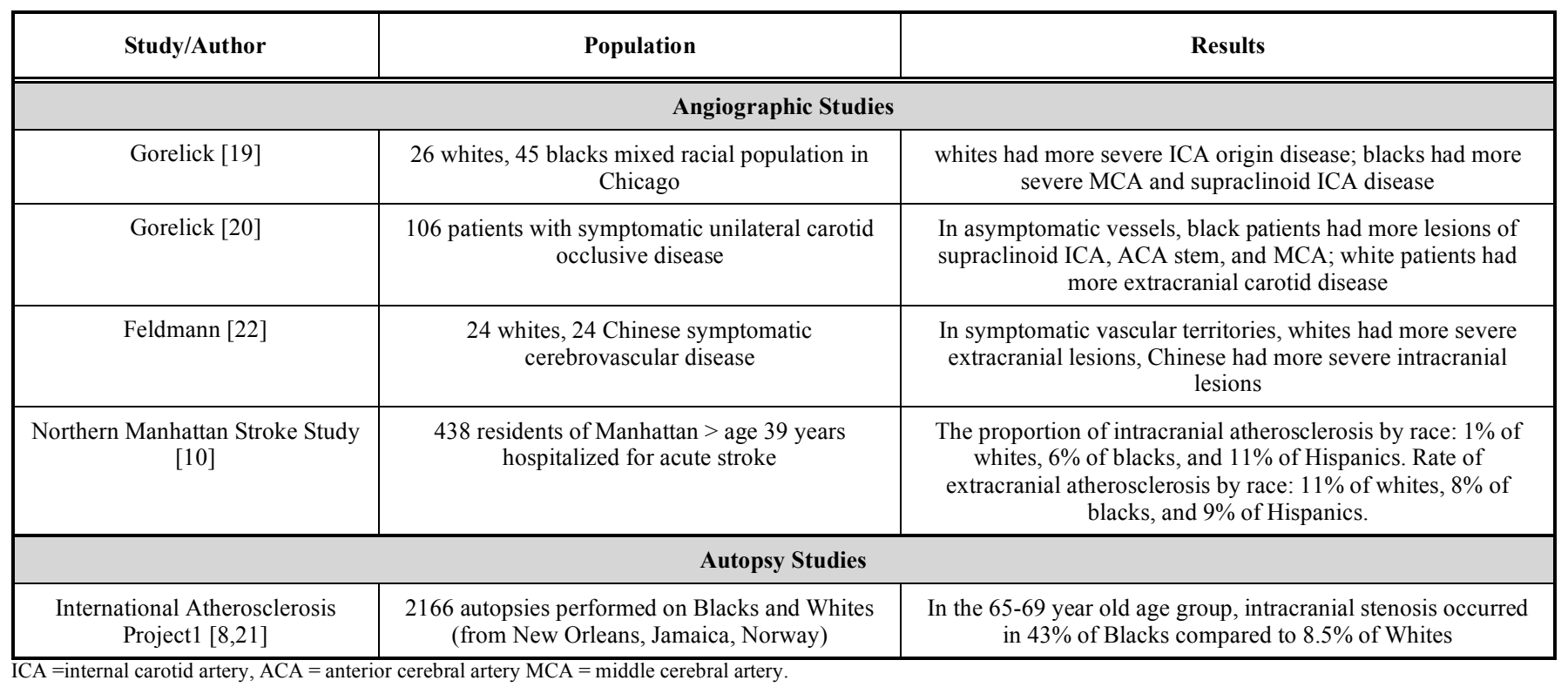


have observed an increased odds ratio of men for developing the disease $[13,21,27,28]$.

\section{Genetic Factors}

Multiple genetic factors have been identified that may enhance and promote premature atherosclerosis in the whole vascular system including intracranial large arteries. These include angiotensin converting enzyme polymorphism, plasma to vascular endothelial growth factor ratio, glutathione S-transferase, omega-1 gene polymorphism, and plasma homocysteine level [13, 29-32]. They exert their action via various mechanisms such as vascular endothelial injury, proliferation of vascular smooth muscle cell, and impairment of angiogenesis.

\section{Modifiable Risk Factors}

\section{Hypertension}

It is the major independent modifiable risk factor for intracranial stenosis. In fact, the impact of hypertension as a risk factor for intracranial atherosclerosis was already established from autopsy studies [18]. Based on epidemiological studies, hypertension is associated with increased odds for the development of intracranial stenosis that ranged from 5 to $9.7[18,27]$. Moreover, the risk further increased when hypertension was associated with other risk factors [7].

\section{Disorders of Lipid Metabolism}

Intracranial stenosis has been associated with dyslipidemia, specifically elevated total cholesterol but also its various components. High lipoprotein is an independent marker for a greater extent of disease. There is actually a synergic effect between lipoprotein "a" and diabetes mellitus and resultant intracranial occlusive disease [12]. Elevated LDL has also been shown to be a risk factor for intracranial stenosis [12].

\section{Diabetes Mellitus}

Diabetes is an independent risk factor for intracranial stenosis. It promotes the accelerated formation of atherosclerotic stenosis through a decrease in fibrinolytic activity $[12,25]$. Based on epidemiological studies, the odds ratio associated with diabetes ranges from 4 to $5.9[12,25$, 27]. In fact, data from the Northern Manhattan Stroke study revealed that patients with intracranial atherosclerosis had a higher prevalence of diabetes $(67 \%)$ when compared to those with extracranial atherosclerosis or non-atherosclerotic $(60 \%$ and $48 \%$ respectively) [33]. Additionally, the impact of diabetes was also established in autopsy study done in Hong Kong [25]. Based on these studies, it may be the strongest risk factor for intracranial stenosis.

\section{Metabolic Syndrome}

Results from the Northern Manhattan Stroke Study [33] also demonstrated a higher prevalence of metabolic syndrome in patient with intracranial atherosclerosis when compared to those with extracranial atherosclerosis, nonatherosclerotic stroke, and controls $(62 \%, 40 \%, 40 \%$, and $35 \%$ respectively).
Other potential though less well-studied factors include sickle cell disease, meningitis, cranial radiation therapy, tobacco exposure, family history, and presence of extracranial carotid atherosclerosis and aortic plaques [13].

\section{Mechanisms and Pathophysiology}

Intracranial arteries are composed of endothelium, smooth muscle cells and an extracellular matrix consisting of collagen and elastin fibers. The luminal tunica intima includes a single layer of endothelial cells overlying delicate connective tissue which is supported by a dense elastic band, the internal elastic lamina. The middle layer, or intima media, consists of smooth muscle cells. The outermost tunica adventitia is mainly composed of collagen and is predominantly surrounded by only cerebrospinal fluid. A cascade of events including macrophage recruitment and low-density lipoprotein accumulation results in atherosclerotic plaque formation.

The several mechanisms of ischemic stroke related to intracranial atherosclerosis include hemodynamic failure, insitu thrombosis from plaque disruption, distal thromboembolism, and perforator artery occlusion by plaque within the parent artery. Perforator artery occlusion seems to be the least frequent mechanism as most subsequent strokes in WASID were non-lacunar (91\%) [34]. An impaired washout concept has also been a proposed mechanism that results from a combination of hypoperfusion and distal thromboembolism [35]. Thus, progressive arterial narrowing, plaque instability and thromboembolism, and/or exhausted collateral flow with impaired vasomotor reactivity are inter-woven mechanisms that may contribute to ischemic stroke due to intracranial stenosis (Table 2).

There is growing evidence for these specific individual mechanisms and their potential synergism [36-40]. Thromboembolic mechanisms include in-situ thrombosis with resultant large artery occlusion, perforator (small artery) occlusion, and/or distal embolism. In one study, among 63 patients with middle cerebral artery stenosis and acute ischemic stroke, 32 showed multiple lesions in the MCA territory [38]. The majority had perforating artery infarcts, alone or in combination with distal territory infarcts while a minority had borderzone infarcts suggesting flow failure. Another study noted that $60 \%$ of patients with multi-infarct pattern on diffusion-weighted imaging had microembolic signals on transcranial Doppler monitoring of the middle cerebral artery compared to only $6 \%$ in those with a single perforator or single infarction pattern [49]. Hemodynamic impairment is likely in a subset of patients with intracranial occlusive disease. With progressive stenosis, the tissue distal to the lesion may depend heavily on collateral blood flow. In one study, $30 \%$ of patients with middle cerebral artery occlusive disease had impaired cerebral hemodynamics on vasomotor reactivity testing [39]. In a small positron emission tomography study, $25 \%$ of patients with symptomatic middle cerebral artery stenosis had abnormal hemodynamics [36]. Using quantitative MRA to estimate vessel-specific flow among those with symptomatic vertebrobasilar stenosis, 16 of 50 patients had impaired flow [37]. 
Table 2. Stroke Mechanism of Large Artery Intracranial Stenosis

\begin{tabular}{|c|c|}
\hline Mechanisms & Imaging Markers (See Chapter 2) \\
\hline \hline Decreased antegrade flow & $\begin{array}{c}\text { Degree of stenosis } \\
\text { Vessel-specific cerebral blood flow }\end{array}$ \\
\hline Plaque instability & Hi-resolution MRI plaque imaging \\
\hline Poor proximal collateral flow & Circle of Willis cerebral blood flow \\
\hline Poor distal collateral flow & Leptomeningeal cerebral blood flow \\
\hline Limited cerebrovascular reserve & Vasomotor reactivity \\
\hline Artery-to-artery embolism & Microembolic signals \\
\hline
\end{tabular}

These data support thromboembolism with local small vessel occlusion and distal embolization as the predominant mechanisms of stroke, though up to one-quarter of patients with severe stenosis or poor collateral flow may develop infarcts due to perfusion failure. It may be that biologic factors that promote plaque changes (i.e. rupture, hemorrhage) and/or a critical level of stenosis must be reached and that leads to a cascade of in-situ events at the level of the atherosclerotic plaque. Antithrombotic agents, statins and elevated blood pressure may favorably influence these events in the setting of acute ischemia.

In clinical practice, the mechanism of stroke due to intracranial stenosis is often inferred by the pattern of infarction on brain imaging, usually magnetic resonance imaging. Deep infarcts suggest local thrombosis at the ostia of perforators. Complete territorial infarction distal to the site of stenosis suggests complete occlusion of the segment. Single or multiple distal cortical infarct patterns suggest thromboembolism from the site of proximal stenosis. Lastly, a borderzone pattern with impaired washout is suggested by "rosary bead" or "string of pearls" appearance in the deep white matter territory of the middle cerebral artery. Often, a mixed or combined pattern is noted which makes definitive determination of stroke mechanism a challenge. A recent case study highlighted how these may interact and provided in vivo evidence of the impaired washout hypothesis [41].

\section{ECONOMIC IMPACT}

The impact of stroke on the individual and on society is enormous. In the US, the costs associated with stroke are estimated to be $\$ 57$ billion annually [2]. A significant portion is due to loss of productivity. Intracranial stenosis alone may be responsible for $\$ 1$ billion annually. Given the increased prevalence of the condition in Asia, the financial and social impact of the disease there may be even greater.

In summary, intracranial stenosis is highly prevalent stroke mechanism, especially in the Asian population that constitute one third of the globe population. Unfortunately, despite the current maximal medical therapy, intracranial stenosis is associated with substantial risk for recurrent stroke and vascular death. Endovascular angioplasty and stent is emerging as a promising alternative modality and its efficacy and safety is currently being assessed in a large multi-center clinical trial. In the mean time, every effort must be made to identify and aggressively control risk factors associated with intracranial atherosclerosis.

\section{REFERENCES}

[1] World Health Organization. The World Health Report 1999 Geneva, WHO, 1999.

[2] American Heart Association. Heart Disease and Stroke Statistics: 2005 Update. Dallas, TX, American Heart Association, 2005.

[3] Thomas GN, Lin JW, Lam WW, et al. Middle cerebral artery stenosis in type II diabetic Chinese patients is associated with conventional risk factors but not with polymorphisms of the renninangiotensin system genes. Cerebrovasc Dis 2003; 16(3): 217-23.

[4] Komotar RJ, Mocco J, Wilson DA, Connolly ES, Lavine SD, Meyers PM. The natural history of intracranial carotid artery atherosclerosis. Neurosurg Focus 2005; 18(1): E4.

[5] Adams HP, Bendixen BH, Kappelle LJ, et al. Classification of subtype of acute ischemic stroke. Definitions for use in a multicenter clinical trial. TOAST. Trial of Org 10172 in Acute Stroke Treatment. Stroke 1993; 24(1): 35-41.

[6] Caplan LR, Gorelick PB, Hier DB. Race, sex and occlusive cerebrovascular disease: a review. Stroke 1986; 17: 648-55.

[7] Wong KS, Ng PW, Tang A, Liu R, Yeung V, Tomlinson B. Prevalence of asymptomatic intracranial atherosclerosis in highrisk patients. Neurology 2007; 68: 2035- 8 .

[8] Savitz SI, Caplan LR. Vertebrobasilar disease. N Engl J Med 2005; 352(25): 2618-26.

[9] Shin HK, Yoo KM, Chang HM, Caplan LR. Bilateral intracranial vertebral artery disease in the New England Medical Center, Posterior Circulation Registry. Arch Neurol 1999; 56(11): 1353-8.

[10] Sacco RL, Kargman DE, Gu Q, Zamanillo MC. Race-ethnicity and determinants of intracranial atherosclerotic cerebral infarction. The Northern Manhattan Stroke Study. Stroke 1995; 26: 14-20.

[11] The Warfarin-Aspirin Symptomatic Intracranial Disease (WASID) Study Group. Prognosis of patients with symptomatic vertebral or basilar artery stenosis. Stroke 1998; 29(7): 1389-92.

[12] Arenillas JF, Molina CA, Chacon P, et al. High lipoprotein (a), diabetes, and the extent of symptomatic intracranial atherosclerosis. Neurology 2004; 63: 27-32.

[13] Suri MK, Johnston SC. Epidemiology of intracranial stenosis. J Neuroimaging 2009; 19: 11S-6S.

[14] Suwanwela NC, Chutinetr A. Risk factors for atherosclerosis of cervicocerebral arteries: intracranial versus extracranial. Neuroepidemiology 2003; 22(1): 37-40.

[15] Wong LKS. Global burden of intracranial atherosclerosis. Int $\mathrm{J}$ Stroke 2006; 1(3): 158-9.

[16] Wong KS, Huang YN, Gao S, Lam WWM, Chan YL, Kay R. Intracranial stenosis in Chinese patients with acute stroke. Neurology 1998; 50: 812-3.

[17] De Silva DA, Woon FP, Lee MP, Chen CP, Chang HM, Wong MC. South Asian patients with ischemic stroke: Intracranial large arteries are the predominant site of disease. Stroke 2007; 38: 25924.

[18] Solberg LA, McGarry PA. Cerebral atherosclerosis in Negroes and Caucasians. Atherosclerosis 1972; 16(2): 141-54.

[19] Gorelick PB, Caplan LR, Hier DB, Parker SL, Patel D. Racial differences in the distribution of anterior circulation occlusive disease. Neurology 1984; 34(1): 54-9.

[20] Gorelick PB, Caplan LR, Langenberg P, et al. Clinical and angiographic comparison of asymptomatic occlusive cerebrovascular disease. Neurology 1988; 38: 852-8. 
[21] Solberg LA, McGarry PA, Moossy J, et al. Distribution of cerebral atherosclerosis by geographic location, race, and sex. Lab Invest 1968; 18: 144-52.

[22] Feldmann E, Daneault N, Kwan E, et al. Chinese-White differences in the distribution of occlusive cerebrovascular disease. Neurology 1990; 40: 1541-5.

[23] Lynch GF, Gorelick PB. Stroke in African Americans. Neurol Clin 2000; 18: 273-90.

[24] Sacco RL, Boden-Albala B, Abel G, et al. Race-ethnic disparities in the impact of stroke risk factors: the northern Manhattan stroke study. Stroke 2001; 32: 1725-31.

[25] Baker AB, Flora GC, Resch JA, et al. The geographic pathology of atherosclerosis: a review of the literature with some personal observations on cerebral atherosclerosis. J Chron Dis 1967; 20: 685-706.

[26] Bae HJ, Lee J, Park JM, et al. Risk factors of intracranial cerebral atherosclerosis among asymptomatics. Cerebrovasc Dis 2007; 24 : 355-60.

[27] Uehara T, Tabuchi M, Hayashi T, Kurogane H, Yamadori A. Asymptomatic occlusive lesions of carotid and intracranial arteries in Japanese patients with ischemic heart disease. Stroke 1996; 27 : 293-7.

[28] Wityk RJ, Lehman D, Klag M, et al. Race and sex differences in the distribution of cerebral atherosclerosis. Stroke 1996; 27: 197480 .

[29] Sertic J, Hebrang D, Janus D, et al. Association between deletion polymorphism of the angiotensin-converting enzyme gene and cerebral atherosclerosis. Eur J Clin Chem Clin Biochem 1996; 34 : 301-4.

[30] Arenillas JF, Alvarez-Sabin J, Montaner J, et al. Angiogenesis in symptomatic intracranial atherosclerosis: predominance of the inhibitor endostatin is related to a greater extent and risk of recurrence. Stroke 2005; 36: 92-7.

[31] Kolsch H, Larionov S, Dedeck O, et al. Association of the glutathione S-transferase omega-1 Ala140Asp polymorphism with cerebrovascular atherosclerosis and plaque-associated interleukin-1 alpha expression. Stroke 2007; 38: 2847-50.
[32] Yoo JH, Chung CS, Kang SS. Relation of plasma homocyst(e)ine to cerebral infarction and cerebral atherosclerosis. Stroke 1998; 29 : 2478-83.

[33] Rincon F, Sacco RL, Kranwinkel G, et al. Incidence and risk factors of intracranial atherosclerotic Stroke: The Northern Manhattan Staoke Study. Cerebrovasc Dis 2009; 28: 65-71.

[34] Famakin BM, Chimowitz MI, Lynn MJ, Stern BJ, George MG. WASID Trial Investigators. Causes and severity of ischemic stroke in patients with symptomatic intracranial arterial stenosis. Stroke 2009; 40(6): 1999-2003.

[35] Caplan LR, Hennerici M. Impaired clearance of emboli (washout) is an important link between hypoperfusion, embolism, and ischemic stroke. Arch Neurol 1998; 55(11): 1475-82.

[36] Derdeyn CP, Powers WJ, Grubb RL Jr. Hemodynamic effects of middle cerebral artery stenosis and occlusion. AJNR Am J Neuroradiol 1998; 19: 1463-9.

[37] Amin-Hanjani S, Du X, Zhao M, Walsh K, Malisch TW, Charbel FT. Use of quantitative magnetic resonance angiography to stratify stroke risk in symptomatic vertebrobasilar disease. Stroke 2005; 36: $1140-45$.

[38] Lee D, Kim J, Kwon S, Yoo S, Kang D. Lesion patterns and stroke mechanism in atherosclerotic middle cerebral artery disease: Early diffusion-weighted imaging study. Stroke 2005; 36: 2583-8.

[39] Ogasawara K, Ogawa A, Yoshimoto T. Cerebrovascular reactivity to acetazolamide and outcome in patients with symptomatic internal carotid or middle cerebral artery occlusion: A xenon-133 single-photon emission computed tomography study. Stroke 2002; 33: $1857-62$.

[40] Wong KS, Gao S, Chan YL, et al. Mechanisms of acute cerebral infarctions in patients with middle cerebral artery stenosis: a diffusion-weighted imaging and microemboli monitoring study. Ann Neurol 2002; 52(1): 74-81.

[41] Schreiber S, Serdaroglu M, Schreiber F, Skalej M, Heinze HJ, Goertler M. Simultaneous occurrence and interaction of hypoperfusion and embolism in a patient with severe middle cerebral artery stenosis. Stroke 2009; 40(7): e478-80.

(C) Mohammad et al.; Licensee Bentham Open.

This is an open access article licensed under the terms of the Creative Commons Attribution Non-Commercial License (http://creativecommons.org/licenses/by$\mathrm{nc} / 3.0 /$ ) which permits unrestricted, non-commercial use, distribution and reproduction in any medium, provided the work is properly cited. 